\title{
Avaliação dos indicadores de um serviço de referência em triagem neonatal no sul do Brasil durante a pandemia por COVID-19.
}

\author{
Evaluation of indicators of a neonatal screening referral service in Southern Brazil during the Covid-19 pandemic. \\ Evaluación de indicadores de um servicio de derivación de tamizaje neonatl em el sur de Brasil durante la pandemia de \\ Covid-19.
}

\section{RESUMO}

Durante a pandemia por COVID-19 foi necessária uma reorganização e um novo planejamento urgente dos fluxos nos Serviços de Triagem Neonatal em todo o Brasil, com o objetivo de minimizar os impactos negativos da pandemia na atenção à saúde da população pediátrica. As principais ações desencadeadas foram a migração das coletas dos exames para as maternidades e a viabilização de consultas à distância evitando a desassistência dos casos confirmados com as doenças triadas. A partir dos novos fluxos, os indicadores de qualidade, como idade no momento da coleta, tempo de transporte das amostras, análise laboratorial e idade dos RN na primeira consulta para confirmação diagnóstica passaram por uma avaliação criteriosa. Objetivo: avaliar o impacto das medidas adotadas num serviço de triagem neonatal antes e durante a pandemia da COVID-19, no Sul do Brasil. Metodologia: estudo transversal serial de base populacional. A coleta de dados foi realizada no banco de dados do Serviço de Referência do estado do RS e na revisão de prontuários. O impacto foi estimado mediante a comparação de indicadores de atendimento do SRTN-RS a partir de setembro de 2019 a maio de 2020. Resultados: o primeiro mês de pandemia apresentou resultados com impacto negativo, mas os números foram restabelecendo-se gradativamente a patamares anteriores à pandemia, conforme novos fluxos foram sendo introduzidos no serviço. Quanto à avaliação no número de consultas, reconvocados e desfechos, foram muito semelhantes para as seis doenças triadas nos períodos pré e pós adoção das medidas de enfrentamento da pandemia, à exceção do Hipotireoidismo Congênito. Os resultados deste estudo mostraram que as rápidas ações de ajustes e adaptações tiveram impacto positivo em manter os bons indicadores de funcionamento da triagem neonatal no programa estadual durante a pandemia da COVID-19.

DESCRITORES: COVID-19, triagem neonatal, fluxos de atendimento, serviço de referência.

\section{ABSTRACT}

During the COVID-19 pandemic, an urgent reorganization and new planning of the flows in Neonatal Screening Services throughout Brazil was necessary, in order to minimize the negative impacts of the pandemic on the health care of the pediatric population. The main actions taken were the migration of exam collections to the maternity hospitals and the feasibility of remote consultations, avoiding the lack of assistance for confirmed cases with the diseases screened for. Based on the new flows, quality indicators, such as age at the time of collection, sample transport time, laboratory analysis and age of the NB at the first appointment for diagnostic confirmation, underwent a careful evaluation. Objective: to evaluate the impact of measures adopted in a neonatal screening service before and during the COVID-19 pandemic in southern Brazil. Methodology: serial cross-sectional population-based study. Data collection was carried out in the database of the Reference Service of the state of RS and in the review of medical records. The impact was estimated by comparing the SRTN-RS service indicators from September 2019 to May 2020 . Results: the first month of the pandemic had results with a negative impact, but the numbers gradually returned to previous levels to the pandemic, as new flows were introduced into the service. Regarding the evaluation of the number of consultations, recalls and outcomes, they were very similar for the six diseases screened in the periods before and after the adoption of measures to fight the pandemic, with the exception of Congenital Hypothyroidism. The results of this study showed that the rapid actions of adjustments and adaptations had a positive impact on maintaining the good functioning indicators of neonatal screening in the state program during the COVID-19 pandemic.

DESCRIPTORS: COVID-19, neonatal screening, care flows, referral service.

\section{RESUMEN}

Durante la pandemia de COVID-19, fue necesaria una reorganización urgente y una nueva planificación de los flujos en los Servicios de Cribado Neonatal en todo Brasil, con el fin de minimizar los impactos negativos de la pandemia en la atención de salud de la población pediátrica. Las principales acciones realizadas fueron la migración de las colecciones de exámenes a las maternidades y la viabilidad de las consultas a distancia, evitando la falta de atención a los casos confirmados con las enfermedades cribadas. A partir de los nuevos flujos, los indicadores de calidad, como la edad en el momento de la recolección, el tiempo de transporte de la muestra, los análisis de laboratorio y la edad del RN en la primera cita para la confirmación 


\section{artigo}

Castro, S. M., Wyzykowski, C., Coutinho, V. L. S., Boianovsky, K., Wiest, P., Kopacek, C.

Avaliação dos indicadores de um serviço de referência em triagem neonatal no sul do Brasil durante a pandemia por COVID-19.

del diagnóstico, fueron sometidos a una cuidadosa evaluación. Objetivo: evaluar el impacto de las medidas adoptadas en un servicio de cribado neonatal antes y durante la pandemia de COVID-19 en el sur de Brasil. Metodología: estudio poblacional transversal seriado. La recogida de datos se realizó en la base de datos del Servicio de Referencia del estado de RS y en la revisión de historias clínicas. El impacto se estimó comparando los indicadores de servicio SRTN-RS de septiembre de 2019 a mayo de 2020. Resultados: el primer mes de la pandemia tuvo resultados con un impacto negativo, pero las cifras regresaron gradualmente a los niveles anteriores a la pandemia, a medida que se introdujeron nuevos flujos. en el servicio. En cuanto a la evaluación del número de consultas, retiros y resultados, fueron muy similares para las seis enfermedades cribadas en los períodos antes y después de la adopción de medidas para combatir la pandemia, a excepción del Hipotiroidismo Congénito. Los resultados de este estudio mostraron que las acciones rápidas de ajustes y adaptaciones tuvieron un impacto positivo en el mantenimiento de los indicadores de buen funcionamiento del cribado neonatal en el programa estatal durante la pandemia de COVID-19.

DESCRIPTORES: COVID-19, cribado neonatal, flujos de atención, servicio de derivación.

RECEBIDO EM : 29/10/2021 APROVADO EM: 06/12/2021

\section{Simone Martins de Castro}

Chefe do Serviço de Referência em Triagem Neonatal, Hospital Materno Infantil Presidente Vargas, Porto Alegre, RS, Professora do Departamento de Farmácia, Universidade Federal do Rio Grande do Sul, Porto Alegre, RS.

ORCID: 0000-0001-6707-9698.

\section{Cintia Wyzykowski}

Enfermeira, Especialista em Pediatria, mestre em enfermagem, doutoranda do Programa de Pós Graduação em Pediatria, Universidade Federal de Ciências da Saúde, Porto Alegre, RS, professora da Universidade Feevale.

ORCID: 0000-0002-5593-4391

\section{Vivian de Lima Spode Coutinho}

Farmacêutica do Serviço de Referência em Triagem Neonatal, Hospital Materno Infantil Presidente Vargas, Porto Alegre, RS, mestranda do Programa de Pós Graduação em Pediatria, Universidade Federal de Ciências da Saúde, Porto Alegre, RS.

ORCID: 0000-0002-3620-2531

\section{Karen Boianovsky}

Médica do Serviço de Referência em Triagem Neonatal, Hospital Materno Infantil Presidente Vargas, Porto Alegre, RS. ORCID: 0000-0002-8679-3085.

\section{Paloma Wiest}

Farmacêutica, Mestranda do Programa de Pós-Graduação em Pediatria, Universidade Federal de Ciências da Saúde, Porto Alegre.

ORCID: 0000-0001-7418-8163.

\section{Cristiane Kopacek}

Médica do Serviço de Referência em Triagem Neonatal, Hospital Materno Infantil Presidente Vargas, Porto Alegre, RS, Professora do Programa de Pós-Graduação em Pediatria, Universidade Federal de Ciências da Saúde, Porto Alegre, RS e Departamento de Pediatria, Universidade Federal de Ciências da Saúde, Porto Alegre, RS.

ORCID: 0000-0001-5229-5443

\section{INTRODUÇÃO}

$\mathbf{0}$ principal objetivo da Triagem Neonatal (TN) é o diagnóstico precoce na fase assintomática, diminuindo morbi-mortalidade. No Brasil, o período ideal de coleta da primeira amostra é a partir das $48 \mathrm{~h}$ até o $5^{\circ}$ dia de vida do bebê, devido às especificidades das condiçôes diagnosticadas, evitando resultados falso-positivos ou falso-negativos. Ressalta-se ainda a importância do gerenciamento dos casos positivos por meio de monitoramento e acompanhamento da criança durante o processo de confirmação diagnóstica e tratamento. Atualmente, no Brasil a triagem pública contempla seis doenças: Hiperplasia Adrenal Congênita (HAC),
Deficiência da Biotinidase (DB), Fibrose Cística (FC), Fenilcetonúria (PKU), Hemoglobinopatias (HB) e Hipotireoidismo Congênito (HC). ${ }^{1}$ Quando não diagnosticadas e tratadas rapidamente, as doenças podem acarretar deficiência intelectual ou, até mesmo, levar ao óbito, impactando diretamente no prognóstico e na qualidade de vida dos infantes afetados ${ }^{1,2}$. 
A restrição de acesso a serviços de saúde e redução de mobilidade, durante a pandemia mundial por COVID-19, atingiu várias políticas de saúde, entre elas os programas de triagem neonatal. Modificações nos tempos de coletas, envios e análises das amostras, bem como a dificuldade de deslocamento para as consultas de atendimento e confirmação diagnóstica no ambulatório especializado foram alguns dos possíveis impactos esperados nos serviços. No entanto, apesar da pandemia, os cuidados com o RN e o diagnóstico precoce não devem sofrer descontinuidade que ocasione o aumento no número de comorbidades e agravos.

Após a instituição das medidas de enfrentamento às ações de contingenciamento, foi necessária uma avaliação criteriosa do funcionamento de toda a rede que envolve a triagem neonatal. O objetivo do presente estudo foi avaliar os indicadores do programa de triagem neonatal, desde a coleta do teste até a confirmação diagnóstica e tratamento, nos períodos anteriores e posteriores à instituição das medidas de enfrentamento da pandemia na primeira onda da COVID-19 no Brasil, em 2020.

\section{MATERIAIS E MÉTODOS}

Este foi um estudo transversal serial de base populacional, realizado no Serviço de Referência em Triagem Neonatal do estado do Rio Grande do Sul (SRTN-RS), localizado no Hospital Materno Infantil Presidente Vargas (HMIPV) do município de Porto Alegre. O Laboratório de Triagem Neonatal recebe amostras de toda a rede pública dos 497 municípios do RS, correspondendo a uma cobertura de $75 \%$ dos nascidos vivos do estado. ${ }^{7}$ As amostras são transportadas principalmente pelos Correios, através de uma remessa expressa. O período do estudo compreendeu seis meses anteriores ao início da pandemia da COVID-19 (setembro/19 a fevereiro/20) e durante os três primeiros meses da primeira onda da pandemia (março a maio de 2020). Os critérios de exclusão adotados foram: pacientes com idade superior a 120 dias na primeira coleta e amostras com dados incompletos, tais como data de coleta, data de nascimento ou peso ao nascer. Foram utilizadas as informações do banco de dados do SRTN-RS (Vega Triagem) e revisão de prontuários. Obteve-se o número total de nascidos vivos através de pesquisa no Sistema de Informações sobre Nascidos Vivos (Sinasc).? Os indicadores analisados foram: percentual de cobertura da primeira coleta e da recoleta; tempo para chegada das amostras de papel-filtro no laboratório; tempo para liberação dos resultados; percentual de reconvocação de novas coletas; média de idade dos RNs na primeira consulta; número de diagnósticos realizados para cada uma das 6 doenças triadas. A reconvocação para coleta de novas amostras foi solicitada pelos seguintes motivos: qualidade da amostra, confirmação de resultados alterados, uso materno de corticoides na última quinzena de gestação e transfusão sanguínea.

As variáveis quantitativas foram descritas por média e desvio padrão ou mediana e amplitude interquartílica. As variáveis categóricas foram descritas por frequências absolutas e relativas. Para avaliar a associação entre as variáveis categóricas, os testes qui-quadrado de Pearson ou exato de Fisher e em caso de variáveis politômicas. A análise dos resíduos ajustados foi utilizada para localizar as associações significativas. Para comparar medianas,

Figura 1. Percentual de cobertura da TN RS e Porto Alegre no Período PréCOVID-19 (setembro a fevereiro) e COVID-19 (março a maio).

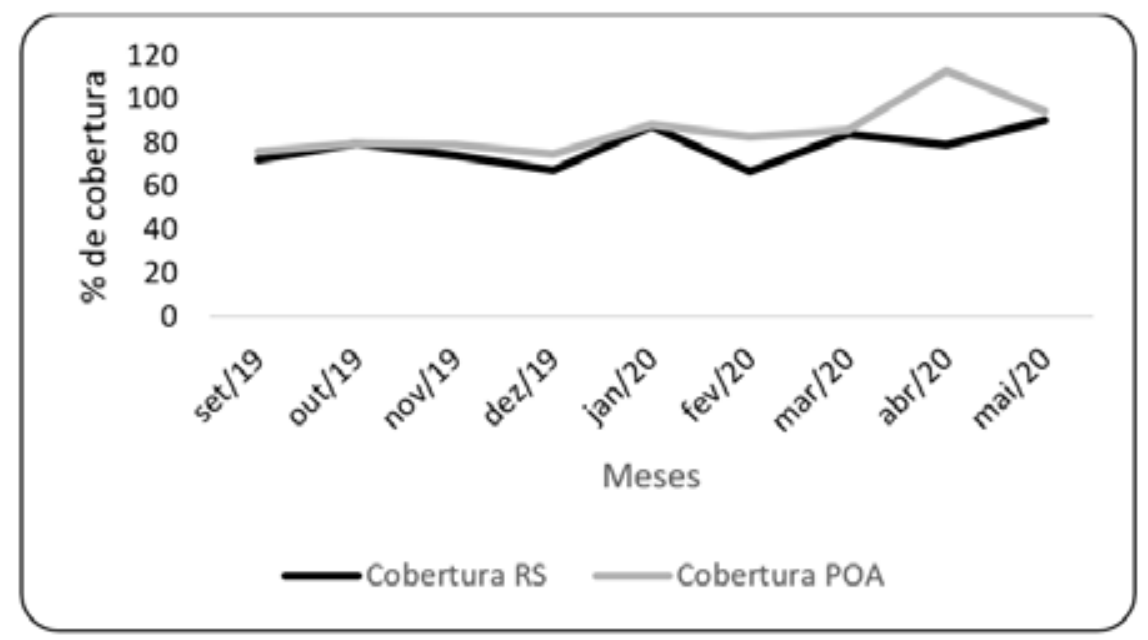

o teste de Mann-Whitney foi aplicado. O nível de significância adotado foi de 5\% $(\mathrm{P}<0,05)$ e as análises foram realizadas no programa SPSS versão 21.0.

\section{RESULTADOS}

De setembro de 2019 a maio de 2020 foram analisados 75.622 pacientes neste estudo. Quanto à cobertura na triagem neonatal não houve diferença significativa no pré e durante a pandemia $(\mathrm{P}=0,167 \mathrm{x}$ $\mathrm{P}=0,048$ ), no estado e município de Porto Alegre (Figura 1). Observamos que no mês de abril houve um acréscimo das coletas na capital, justificado pelo aumento das coletas nas maternidades.

A Figura 2 apresenta o percentual de coletas de 0 a 2 e de 3 a 5 dias no estado e na capital. De março a maio, houve um aumento de coletas precoces $(\mathrm{P}<0,05)$, comparado aos meses que antecederam a

A Tabela 1 demonstra o possível impacto das ações de enfrentamento ao contingenciamento imposto pela pandemia no número de reconvocados, no número de desfechos e o número de consultas para os casos suspeitos. As estratégias adotadas não impactaram no aumento do percentual de reconvocações durante o período de pandemia estudado. pandemia da COVID-19. 


\section{artigo}

Castro, S. M., Wyzykowski, C., Coutinho, V. L. S., Boianovsky, K., Wiest, P., Kopacek, C.

Avaliação dos indicadores de um serviço de referência em triagem neonatal no sul do Brasil durante a pandemia por CovID-19.

Figura 2. Figura (A) representa o percentual do número de coletas de $0-2$ dias de vida do $R N$ no $R S(P=0,024)$ e em Porto Alegre ( $P=0,024)$. A Figura $B$ representa o percentual do número de coletas de 3-5 dias de vida do RN no RS $(P=0,024)$ e em Porto Alegre $(P=0,024)$.

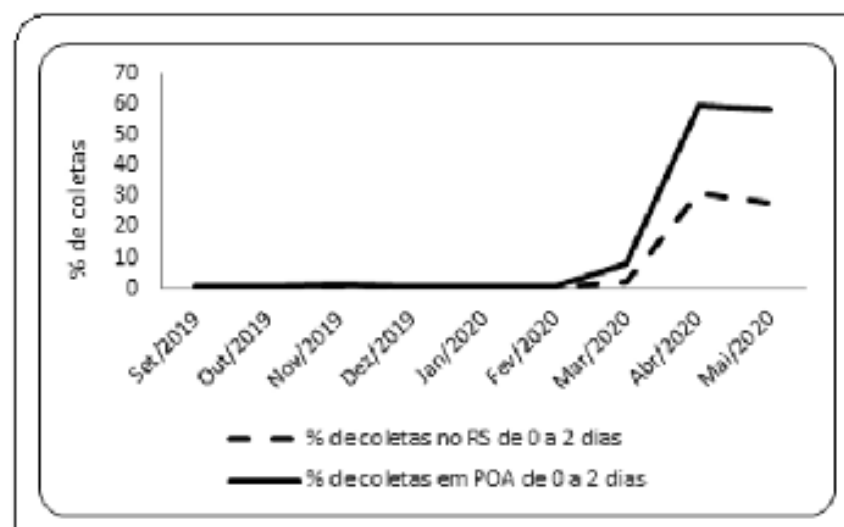

Figura A

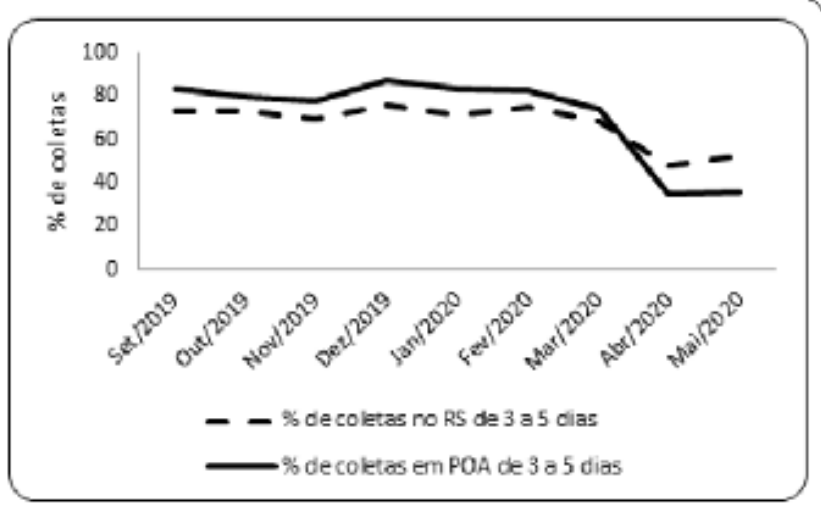

Figura B
Entre as doenças, o maior impacto foi observado na triagem do HC. Os níveis de TSH neonatal possuem valores diferenciados de acordo com a idade no momento da coleta. As coletas precoces levaram a um aumento dos resultados falso-positivos e do número de consultas no início da pandemia. Para a HAC, houve uma diminuição $(\mathrm{P}<0,05)$ nos números de consultas e de reconvocados e um aumento $(\mathrm{P}<0,05)$ no número de desfechos falso-positivos. Contudo, o número de doentes nas fases pré-COVID-19 e COVID-19 foram iguais. Em relação à DB, o único parâmetro que apresentou uma diminuição $(P<0,05)$, foi o desfecho falso-positivo no período COVID-19, denotando assim, que os ajustes realizados não impactaram de forma negativa nesta doença. $\mathrm{Na}$ triagem de $\mathrm{FC}, \mathrm{HB}$ e $\mathrm{PKU}$ não foram observadas diferenças estatísticas

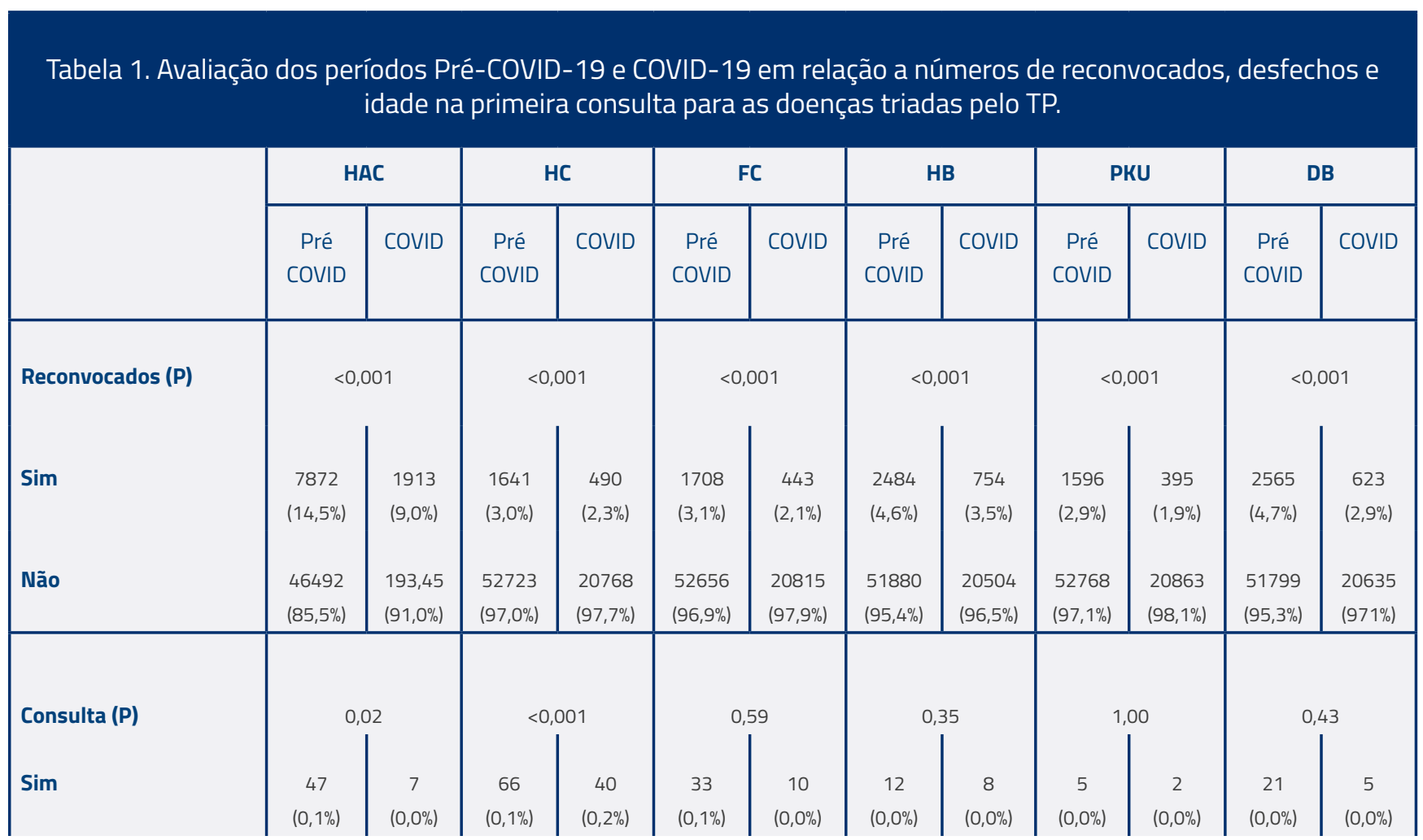




\begin{tabular}{|c|c|c|c|c|c|c|c|c|c|c|c|c|}
\hline Não & $\begin{array}{l}54317 \\
(99,9 \%)\end{array}$ & $\begin{array}{c}21251 \\
(100,0 \%)\end{array}$ & $\begin{array}{l}54298 \\
(99,9 \%)\end{array}$ & $\begin{array}{l}21218 \\
(99,8 \%)\end{array}$ & $\begin{array}{l}54331 \\
(99,9 \%)\end{array}$ & $\begin{array}{c}21248 \\
(100,0 \%)\end{array}$ & $\begin{array}{c}54352 \\
(100,0 \%)\end{array}$ & $\begin{array}{c}21250 \\
(100,0 \%)\end{array}$ & $\begin{array}{c}54359 \\
(100,0 \%)\end{array}$ & $\begin{array}{c}21256 \\
(100,0 \%)\end{array}$ & $\begin{array}{c}54343 \\
(100,0 \%)\end{array}$ & $\begin{array}{c}21253 \\
(100,0 \%)\end{array}$ \\
\hline Desfecho (P) & \multicolumn{2}{|c|}{$<0,001$} & \multicolumn{2}{|c|}{$<0,001$} & \multicolumn{2}{|c|}{0,378} & \multicolumn{2}{|c|}{0,572} & \multicolumn{2}{|c|}{0,723} & \multicolumn{2}{|c|}{$<0,001$} \\
\hline Verdadeiro positivo & $\begin{array}{c}1 \\
(0,0 \%)\end{array}$ & $\begin{array}{c}1 \\
(0,0 \%)\end{array}$ & $\begin{array}{c}43 \\
(0,1 \%)\end{array}$ & $\begin{array}{c}10 \\
(0,0 \%)\end{array}$ & $\begin{array}{c}4 \\
(0,0 \%)\end{array}$ & $\begin{array}{c}3 \\
(0,0 \%)\end{array}$ & $\begin{array}{c}8 \\
(0,0 \%)\end{array}$ & $\begin{array}{c}6 \\
(0,0 \%)\end{array}$ & $\begin{array}{c}2 \\
(0,0 \%)\end{array}$ & $\begin{array}{c}0 \\
(0,0 \%)\end{array}$ & $\begin{array}{c}4 \\
(0,0 \%)\end{array}$ & $\begin{array}{c}4 \\
(0,0 \%)\end{array}$ \\
\hline Verdadeiro negativo & $\begin{array}{l}54077^{*} \\
(99,5 \%)\end{array}$ & $\begin{array}{l}20795 \\
(97,8 \%)\end{array}$ & $\begin{array}{l}54298^{*} \\
(99,9 \%)\end{array}$ & $\begin{array}{l}21193 \\
(99,7 \%)\end{array}$ & $\begin{array}{l}53947 \\
(99,2 \%)\end{array}$ & $\begin{array}{l}53947 \\
(99,2 \%)\end{array}$ & $\begin{array}{l}21118 \\
(99,3 \%)\end{array}$ & $\begin{array}{l}54028 \\
(99,4 \%)\end{array}$ & $\begin{array}{l}21128 \\
(99,4 \%)\end{array}$ & $\begin{array}{l}54062 \\
(99,4 \%)\end{array}$ & $\begin{array}{l}54065 \\
(99,5 \%)\end{array}$ & $\begin{array}{l}21179 * \\
(99,0 \%)\end{array}$ \\
\hline Falso positivo & $\begin{array}{c}267 \\
(0,5 \%)\end{array}$ & $\begin{array}{c}459^{*} \\
(2,2 \%)\end{array}$ & $\begin{array}{c}14 \\
(0,0 \%)\end{array}$ & $\begin{array}{c}52^{*} \\
(0,2 \%)\end{array}$ & $\begin{array}{c}122 \\
(0,2 \%)\end{array}$ & $\begin{array}{c}44 \\
(0,2 \%)\end{array}$ & $\begin{array}{c}3 \\
(0,0 \%)\end{array}$ & $\begin{array}{c}2 \\
(0,0 \%)\end{array}$ & $\begin{array}{c}27 \\
(0,0 \%)\end{array}$ & $\begin{array}{c}11 \\
(0,1 \%)\end{array}$ & $\begin{array}{c}256^{*} \\
(0,5 \%)\end{array}$ & $\begin{array}{c}47 \\
(0,2 \%)\end{array}$ \\
\hline Incompleto & $\begin{array}{c}19 \\
(0,0 \%)\end{array}$ & $\begin{array}{c}3 \\
(0,0 \%)\end{array}$ & $\begin{array}{c}9 \\
(0,0 \%)\end{array}$ & $\begin{array}{c}3 \\
(0,0 \%)\end{array}$ & & & $\begin{array}{c}325 \\
(0,6 \%)\end{array}$ & $\begin{array}{l}1229 \\
(0,6 \%)\end{array}$ & $\begin{array}{c}273 \\
(0,5 \%)\end{array}$ & $\begin{array}{c}98 \\
(0,5 \%)\end{array}$ & $\begin{array}{c}39 \\
(0,1 \%)\end{array}$ & $\begin{array}{c}28 \\
(0,1 \%)\end{array}$ \\
\hline
\end{tabular}

nos parâmetros analisados entre o período pré-COVID-19 e durante COVID-19.

\section{DISCUSSÃO}

A TN é um importante programa de saúde pública. Entre as diversas políticas de saúde da criança, avaliar e qualificar os indicadores da triagem neonatal durante a pandemia é fundamental. Com o distanciamento social imposto, o fechamento de inúmeras USs e a redução dos profissionais no atendimento na atenção básica havia a expectativa de uma redução na cobertura da TN e nos diagnósticos, que não foram observados em nosso estudo. Visando minimizar o impacto negativo da pandemia, os serviços seguiram a orientação do MS, autorizando as coletas nas maternidades, preconizadas para acima de $48 \mathrm{~h}$ de vida do RN. ${ }^{3,4}$ Essa orientação acarretou uma reestruturação nos fluxos do SRTN, de modo a garantir a cobertura e a qualidade do serviço, no que diz respeito ao monitoramento de coletas precoces. Semelhante a um estudo realizado na Austrália, os autores ressaltam que no período inicial da pandemia, em 2020, foi necessário traçar estratégias rapidamente, para dar conti- nuidade ao serviço de laboratório e assim o impacto no serviço não ser negativo. Isso permitiu manter o serviço sem efeitos perceptíveis em sua qualidade, apesar dos desafios de se adaptar rapidamente a novos processos de trabalho e trabalhar dentro das restrições do governo. Os autores ainda destacam que a prontidão e agilidade de implementação foram baseadas com foco no recém-nascido e na família, no sistema de produção e uma mentalidade de melhoria contínua ${ }^{5}$.

Nossos resultados também demonstraram que, mesmo com as medidas emergenciais adotadas, a autonomia dos gestores de cada município em conjunto com o serviços de enfermagem, responsáveis por buscas ativas e tantos outros programas de saúde, permitiu preconizar e manter o olhar global na saúde da criança, uma vez que existem outros programas importantes nesse contexto, tais como vacinas, aleitamento materno, puericultura também de extrema importância e que necessitavam ser mantidos. ${ }^{7,8}$ Assim, foram instituídas, nesse período, estratégias de saúde municipais que priorizaram visitas domiciliares que atendessem aos programas de saúde da criança e da mulher, seguindo com coletas em tempo ideal, sem impacto na cobertura do programa de $\mathrm{TN} .{ }^{10}$

Devido à situação de distanciamento social, uma alternativa foi o alavancamento da modalidade de tele-atendimento. Recentemente foi regulamentada pelo Ministério da Saúde, Conselho Federal de Medicina e Conselho Federal de Psicologia. Orientações nesse sentido foram publicadas pela Cruz Vermelha Internacional e pelo Centro de Estudos e Pesquisas em Emergências e Desastres em Saúde. ${ }^{11,12,13,14}$ Tal alternativa permitiu agilidade no contato com equipes de saúde dos municípios para casos suspeitos e rápidas providências para confirmar diagnósticos e instituir tratamentos. Schwamm (2014) ressalta que a telessaúde é a oferta de serviços de saúde mediada por tecnologia. Pode ser estrategicamente vista como uma inovação disruptiva que coloca em xeque as práticas tradicionais de saúde, com redução de custos e ganhos na qualidade, no acesso e na satisfação do usuário. ${ }^{15}$ Corroborando com isso, cabe destaque, então, para a Portaria 467 do Ministério da Saúde, que autoriza a prática da telemedicina, para atender pacientes em meio à pandemia atual, à distância. ${ }^{11} \mathrm{~A}$ partir dessa perspectiva, 
Greenhalgh comenta que as modalidades de atendimento on-line têm sido priorizadas e bastante difundidas, o que impóe a necessidade de se estender o acesso à telefonia celular e Internet de forma rápida para profissionais e usuários. A consulta remota deve ser realizada com base em protocolos, com mensagens claras e objetivas, dando preferência ao vídeo. ${ }^{16}$ Assim, é possível fazer o atendimento de qualidade à distância, orientando e capacitando equipes de saúde locais, evitando deslocamentos desnecessários e minimizando as chances de exposição ao vírus, no contexto atual. Dessa forma, a tele-consulta se revela como uma ação de enfrentamento bastante eficaz, que pode contribuir também no acompanhamento de maior parte da população, evitando a aglomeração dos campos hospitalares onde há grande risco de contágio. ${ }^{16}$

Cada uma das seis doenças triadas possui quadro clínico peculiar e especificidades para a triagem, o que requer fluxos e olhares individualizados. ${ }^{1}$ Para o diagnóstico de PKU, as coletas precoces podem impactar sensivelmente no desfecho, visto que a fenilalanina é gerada pela metabolização de proteínas ingeridas após o nascimento. Caso a coleta seja realizada antes deste processo, resultados falso-negativos podem acontecer. ${ }^{1,17,18,19}$ Apesar das coletas precoces apresentarem um risco para diagnóstico de $\mathrm{PKU}$, não foram observadas diferenças estatísticas nos dois períodos avaliados quanto às consultas, desfechos e percentual de reconvocados. Da mesma forma, mesmo com o aumento no tempo de transporte das amostras, não houve impacto nos parâmetros analisados para a DB, visto que a atividade da biotinidase decai sensivelmente com a ação de altas temperaturas e umidade. ${ }^{20}$ Já no caso da HAC, o diagnóstico precoce é crucial para prevenir o óbito de lactentes por insuficiência adrenal. Os programas de triagem para HAC visam, principalmente, o diagnóstico precoce da forma perdedora de sal, mais grave e potencialmente letal.6,21 Apesar de ter sido evidenciada a diminuição no número de consultas para HAC durante a pandemia, o número de

\section{Cada uma das seis} doenças triadas

possui quadro clínico peculiar e especificidades

para a triagem, o que requer

fluxos e olhares individualizados. ${ }^{1}$

Para o diagnóstico de PKU, as coletas precoces podem impactar sensivelmente no desfecho, visto que a fenilalanina é gerada pela metabolização de proteínas ingeridas após o nascimento doentes nas fases pré-COVID-19 e COVID-19 foram semelhantes. Atribui-se tal resultado à agilidade da equipe em buscar informações clínicas e resultados de recoletas dos bebês suspeitos e desta forma rapidamente concluir sobre o desfecho e orientar as equipes locais.

Com relação ao $\mathrm{HC}$, a coleta nas maternidades causou impacto no número de consultas e no percentual de falsos-positivos. Os valores do hormônio TSH é mais elevado nas primeiras 48 horas de vida do RN.22,23,24,25 Com o aumento das coletas precoces, o número de resultados falsamente elevados gerou um número maior de consultas, até que o serviço estabelecesse novos valores de referência para este teste no período de 0 a $48 \mathrm{~h}$ de vida do RN. Não houve influência no número de reconvocados, pois a estratégia inicial foi encaminhar os pacientes suspeitos diretamente para a consulta sem recoletas, no intuito de agilizar o processo de diagnóstico, uma vez que o $\mathrm{HC}$ requer o início do tratamento até o $15^{\circ}$ dia de vida do bebê para evitar sequelas neurológicas.22,23,24,25

Apesar do contigenciamento imposto pela pandemia, houve a participação ativa das Secretarias Municipais de Saúde (SMS), mantendo os fluxos e programas visando à saúde integral das crianças. Foram adotadas medidas que, efetivamente, colaboraram para o alcance dos propósitos do programa, entre elas coletas domiciliares, reforçando a necessidade de redes de colaboração e tudo isso, com o trabalho conjunto da equipe de enfermagem e toda equipe multiprofissional.

As medidas de contingenciamento afetaram significativamente os fluxos de transporte, com o fechamento de agências de Correios, restrições de mobilidade intermunicipal e redução de equipes de saúde, os meios de traslado das amostras da TN foram prejudicados. Passado o momento mais delicado e inicial da pandemia, foi observada uma retomada progressiva nos fluxos normais de atendimento das USs e nos sistemas de transporte estadual e municipal. Nessa perspectiva, Aquino et al reforçam, que a reorganização dos serviços de Atenção Primária à 
Saúde (APS) para, simultaneamente, enfrentar a epidemia e manter a oferta regular de suas ações é imperativa.26 Também, as atividades de rotina da APS precisam ser preservadas em tempos de pandemia, especialmente se considerarmos que as previsões apontam para um longo curso de convivência com o novo vírus, com alternância de maior e menor isolamento social, o que exige readequação de certos procedimentos, incluindo novas formas de cuidado cotidiano à distância, evitando o risco de aprofundamento da exclusão do acesso e das desigualdades sociais. 27

\section{CONCLUSÃO}

O período inicial da pandemia da COVID-19 gerou muitas ações restritivas que poderiam impactar de forma significativa no funcionamento adequado do SRTN. Mas com o presente estudo, observou-se que, com a reorganização rápida da rede $\mathrm{e}$ mudanças de fluxos, não houve um grande impacto no Serviço. Além disso, a implantação da telemedicina estreitou relações com as equipes médicas da Atenção Básica dos municípios para a discussão dos casos suspeitos e em fase de diagnóstico com o intuito de qualificá-los para o atendimento inicial desses bebês. O Serviço continuará avaliando, até o final da pandemia, possíveis modificações nos indicadores de qualidade, para que, se necessário, medidas rápidas e eficientes sejam tomadas, evitando qualquer impacto negativo no diagnóstico e na assistência da TN.

\section{APROVAÇÃO ÉTICA:}

O projeto foi aprovado pelos Comitês de Ética e Pesquisa (CEP) CAAE: 32217520.8.0000.5345 e do HMIPV

CAAE: 32217520.8.3001.5329, da Universidade Federal de Ciências da Saúde de Porto Alegre (UFCSPA).

\section{REFERÊNCIAS}

1.Brasill Ministério da Saúde. Triagem Neonatal Biológica - Manual Técnico. 2016.https://bvsms.saude.gov.br/bvs/publicacoes/ triagem_neonatal_biologica_manual_tecnico.pdf (09 november 2020, date last accessed).

2.Rodrigues LP, Vaz Tanaka SCS, Haas VJ, Cunali VCA, De Marqui ABT. Heel prick test: Maternal-fetal conditions that may have an effect on the test results in newborns admitted to the intensive care unit. Rev Bras Ter Intensiva. 2019; 31(2):186-92.

3.Conselho Nacional de Saúde (CNS). Recomendação no 022, de 09 de abril de 2020.http://conselho.saude.gov.br/recomendacoes-cns/1112-recomendac-a-o-n-022-de-09-de-abrilde-2020 (09 november 2020, date last accessed).

4.Brasil Ministério da Saúde. Nota informativa no 4/2020. 2020. https://antigo.saude.gov.br/images/pdf/2020/marco/31/Nota-Informativa-4-PNTN-25000.040288_2020_38.pdf (09 november 2020, date last accessed).

5.Greaves, RF, Pitt, J., McGregor, C. et al. Newborn bloodspot screening in the time of COVID-19. Genet Med 23, 1143-1150 (2021). https://doi.org/10.1038/s41436-020-01086-6

6.Kopacek C, de Castro SM, Prado MJ, da Silva CMD, Beltrão LA, Spritzer PM. Neonatal screening for congenital adrenal hyperplasia in Southern Brazil: A population based study with 108,409 infants. BMC Pediatr. 2017;17(1):1-7.

7.Sistema de informação sobre Nascidos Vivos - SINASC. http:// sinasc.saude.gov.br/default.asp. (09 november 2020, date last accessed).

8.Brasil Ministério da Saúde. Portaria N 1.130, de 5 de agosto de 2015. 2015. http://bvsms.saude.gov.br/bvs/saudelegis/ gm/2015/prt1130_05_08_2015.html (09 november 2020, date last accessed).

9. Brasil Ministério da Saúde. Política Nacional de Atenção Integral à Saúde da Criança - Orientações para Implementação. 2018.https://data.dre.pt/eli/port/141/2018/05/18/p/dre/pt/ htm (09 november 2020, date last accessed).

10. Brasil Ministério da Saúde. Agenda de compromissos para a saúde integral da criança e redução da mortalidade infantil. 2004. http://bvsms.saude.gov.br/bvs/publicacoes/agenda_ compro_crianca.pdf (09 november 2020, date last accessed).

11. ABRASCO - Associação Brasileira de Saúde Coletiva. Desafios da APS no SUS no enfrentamento da Covid-19. 2020. http:// www.abeno.org.br/arquivos/downloads/ABRASCO.pdf (09 november 2020, date last accessed).

12.Brasil Ministério da Saúde. Portaria N467, de 20 de março de 2020. 2020. https://www.in.gov.br/en/web/dou/-/portarian-467-de-20-de-marco-de-2020-249312996 (09 november 2020, date last accessed).

13.Conselho Federal de Medicina - CFM. Telemedicina: CFM regulamenta atendimentos online no Brasil. 2019. http://www. portal.cfm.org.br/index.php?option=com_content\&view=article\&id=28061 (09 november 2020, date last accessed).

14.Conselho Federal de Psicologia - CFP. Coronavírus : Comunicado sobre atendimento on-line. 2020. https://site.cfp.org.br/ coronavirus-comunicado-sobre-atendimento-on-line/ (09 november 2020, date last accessed).

15.Lima RC. Distanciamento e isolamento sociais pela COVID-19 no Brasil: Impactos na saúde mental. Physis. 2020;30(2):1-10. 


\section{REFERÊNCIAS}

16.Schwamm LH. Telehealth: Seven strategies to successfully implement disruptive technology and transform health care. Health Aff. 2014;33(2):200-6.

17.Greenhalgh T, Koh GCH, Car J. Covid-19: A remote assessment in primary care. BMJ. 2020;368:1-5.

18.Botler J, Camacho LAB, Cruz MM da. Phenylketonuria, congenital hypothyroidism and haemoglobinopathies: public health issues for a Brazilian newborn screening program. Cad Saude Publica. 2012;28(9):1623-31.

19.Mira NV de, Marquez UML. Importância do diagnóstico e tratamento da fenilcetonúria. Rev Saude Publica. 2000;34(1):86-96.

20.Stranieri I, Takano OA. Evaluation of the neonatal screening program for congenital hypothyroidism and phenylketonuria in the State of Mato Grosso, Brazil. Arq Bras Endocrinol Metabol. 2009;53(4):446-52.

21.Borsatto T, Sperb-Ludwig F, Blom HJ, Schwartz I V.D. Effect of BTD gene variants on in vitro biotinidase activity.Mol Genet Metab. 2019 Aug;127(4):361-7.

22.Kopacek C, Castro SM, Chapper M, Amorim LB, Lüdtke C, Var- gas P. Evolução e funcionamento do Programa Nacional de Triagem Neonatal no Rio Grande do Sul de 2001 a 2015. Bol Científico Pediatr. 2015;04(3):70-4.

23.Ramalho ARO, Ramalho RJR, Oliveira CRP, Santos EG, OIiveira MCP, Aguiar-Oliveira MH. Programa de Triagem Neonatal para Hipotireoidismo Congênito no nordeste do Brasil: Critérios diagnósticos e resultados. Arq Bras Endocrinol Metabol. 2008;52(4):617-27.

24.Weiner A, Oberfield S, Vuguin P. The laboratory features of congenital hypothyroidism and approach to therapy. Neoreviews. 2020;21(1):e37-44.

25.Eng $L$, Lam $L$. Thyroid function during the fetal and neonatal periods. Neoreviews. 2020;21(1):e30-6.

26.Kaplowitz PB. Neonatal Thyroid Disease: Testing and Management. Pediatr Clin North Am. 2019;66(2):343-52.

27.Aquino EML, Silveira IH, Pescarini JM, Aquino R, de Souza-Filho JA. Social distancing measures to control the COVID-19 pandemic: Potential impacts and challenges in Brazil. Cienc e Saude Coletiva. 2020;25:2423-46. 